\title{
OPTOELECTRONIC STUDIES IN NANOCRYSTALLINE SILICON SCHOTTKY DIODES OBTAINED BY HOT-WIRE CVD
}

\author{
C.Voz, ${ }^{1}$ D.Soler, ${ }^{2}$ M.Fonrodona, ${ }^{2}$ J.Bertomeu, ${ }^{2}$ J.M.Asensi ${ }^{2}$ and J.Andreu ${ }^{2 *}$ \\ ${ }^{1}$ Departament d'Enginyeria Electrònica, Universitat Politècnica de Catalunya \\ Av. Víctor Balaguer s/n, E-08800, Vilanova i la Geltrú (Spain) \\ ${ }^{2}$ Departament de Física Aplicada i Òptica, Universitat de Barcelona \\ Av. Diagonal 647, E-08028 Barcelona (Spain)
}

\begin{abstract}
The very usual columnar growth of nanocrystalline silicon leads to electronic transport anisotropies. Whereas electrical measurements with coplanar electrodes only provide information about the electronic transport parallel to the substrate, it is the transverse transport which determines the collection efficiency in thin film solar cells. Hence, Schottky diodes on transparent electrodes were obtained by Hot-Wire CVD in order to perform external quantum efficiency and surface photovoltage studies in sandwich configuration. These measurements allowed to calculate a transverse collection length which must correlate with the photovoltaic performance of thin film solar cells. Furthermore, the density of charge trapped at localised states in the bandgap was estimated from the voltage dependence of the depletion capacitance of these rectifying contacts.
\end{abstract}

Keywords: nanocrystalline silicon, Hot-Wire CVD, electronic transport, solar cell

\section{Introduction}

Recently, hydrogenated nanocrystalline silicon (nc-Si:H) has attracted great attention as a new promising material for thin film photovoltaic devices. This kind of small-grain silicon allows to combine the low temperature deposition techniques known from the amorphous silicon technology with the long term stability of crystalline silicon. To date, the Hot-Wire CVD (HWCVD) technique allows the highest deposition rates in the range of some tens of $\AA / \mathrm{s}$. Encouraging results have been obtained by this technique at the Debye Institute of University of Utrecht with a $4.4 \%$ efficiency solar cell only $1.2 \mu \mathrm{m}$ thick on stainless steel[1]. In the last years, the group at University of Barcelona is also investigating the possibilities of HWCVD for obtaining device grade nc-Si:H at

${ }^{*}$ Corresponding author: tel: +34 9340211 45; fax: +34 9340211 38; e-mail: jandreu@fao.ub.es 
low substrate temperatures $\left(<300^{\circ} \mathrm{C}\right)$. The usual columnar growth observed in most nc-Si:H layers results in anisotropic electronic transport as previously reported by other groups[2]. While usual electrical measurements are performed with coplanar electrodes, collection in thin film solar cells is perpendicular to the plane of the active layer. In order to get more insight into the transverse electronic transport, measurements with electrodes in sandwich configuration would be required.

\section{Experimental}

Undoped nc-Si:H films were deposited by HWCVD on Corning 7059, c-Si wafer (111) and $\mathrm{ZnO}$ :Al-coated glass in one of the chambers of an UHV multichamber set-up described elsewhere[3]. A tungsten wire, heated to above $1600{ }^{\circ} \mathrm{C}$ by the Joule effect, catalytically decomposes the gas mixture (silane and hydrogen) during the deposition. The $\mathrm{ZnO}$ :Al/undoped nc$\mathrm{Si}: \mathrm{H}$ contacts showed a clear rectifying behaviour. The depletion capacitance of these Schottky contacts was obtained with an impedance analyser. From its voltage dependence at low frequencies $(<1 \mathrm{KHz})$ we estimated the density of charged centres within the depletion region[4]. The shortcircuit external quantum efficiency was measured by means of a grating monochromator, a mechanical chopper $(<10 \mathrm{~Hz})$ and a lock-in amplifier. Besides, the open-circuit surface photovoltage, which allows to deduce an effective transverse diffusion length[5], was also measured with the same experimental set-up. In both studies, the structure was illuminated through the glass/ZnO:Al transparent electrode. Additionally, the optical absorption coefficient was measured by photothermal deflection spectrometry (PDS) and the crystalline fraction of the samples was evaluated by Raman spectroscopy (results not shown in this work).

\section{Results and discussion}

We have recently obtained a clear enhancement in the photovoltaic performance of our $\mathrm{p}-\mathrm{i}-\mathrm{n}$ solar cells by increasing the deposition rate of the undoped active layer[6]. By contrast, structural characterisations pointed to a similar microstructure and crystalline fraction. Neither significant differences were observed in the subgap optical absorption measured by PDS. Hence, in order to guess the origin of the clearly improved collection, we compared several Schottky contacts grown at clearly different deposition rates. Two structures representative for the overall trend are shown in this work. The Schottky contact $A$, obtained at a high deposition rate of $11.3 \AA$ As, is compared to the $B$ contact, which was grown at a much lower deposition rate of only $2.2 \AA / s$. The density of charged centres was calculated by fitting the voltage dependence of the depletion capacitance (Fig. 1) according to the well-known equation

$$
\frac{1}{C^{2}}=\frac{2}{e \varepsilon_{s} N_{c}}\left(V_{d}-V\right)
$$

where $e$ is the elementary charge, $C$ the depletion capacitance per unit area, $\varepsilon_{s}$ the dielectric permitivity of silicon, $V_{d}$ the Schottky barrier and $N_{c}$ the concentration of charged centres within the depletion region of width $w$. The Schottky contact $A$, which was grown faster, showed a clearly reduced density of trapped charge which points to a lower concentration of localised states in the bandgap and consequently a wider depletion region. 


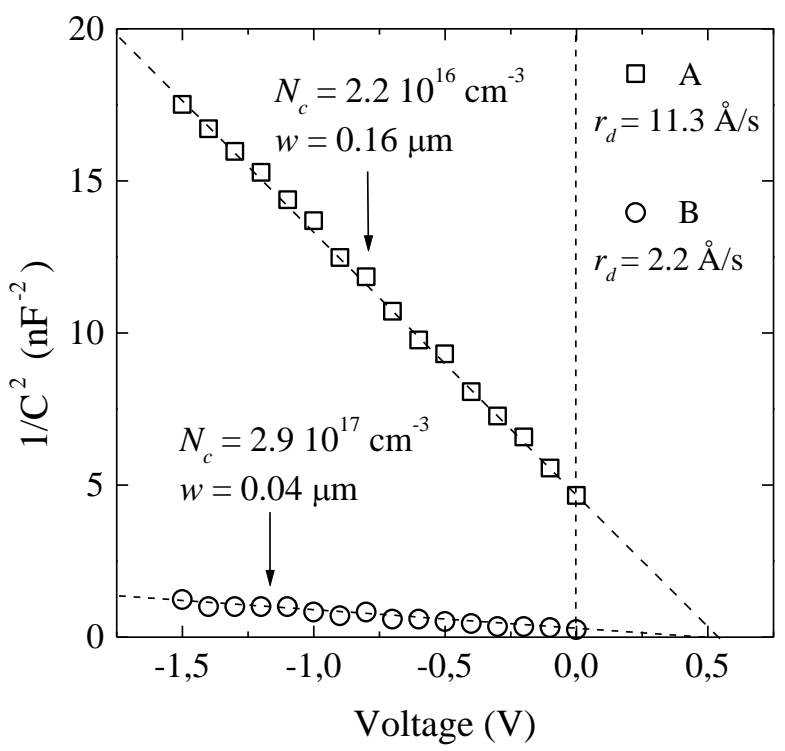

Fig. 1. Determination of the concentration of charged centres $N_{c}$ from the voltage dependence of the depletion capacitance of the Schottky contacts $A$ and $B$ obtained at different deposition rates. As expected, the width of the depletion region $w$ is inversely proportional to the square root of $N_{c}$.

The subgap optical absorption of these samples measured by PDS did not correlate with the calculated values of $N_{c}$. Furthermore, according to reported calibrations with electron-spin resonance measurements[7], the density of deep states (dangling bonds) was lower than $10^{16} \mathrm{~cm}^{-3}$ for both samples. Therefore, calculated densities of charged centres in the order of $10^{17} \mathrm{~cm}^{-3}$, as in the $B$ Schottky contact, must be due to states not resolvable by subgap optical absorption due to its shallow position in the gap. We can attribute the origin of these levels to the undesired incorporation of contaminants.
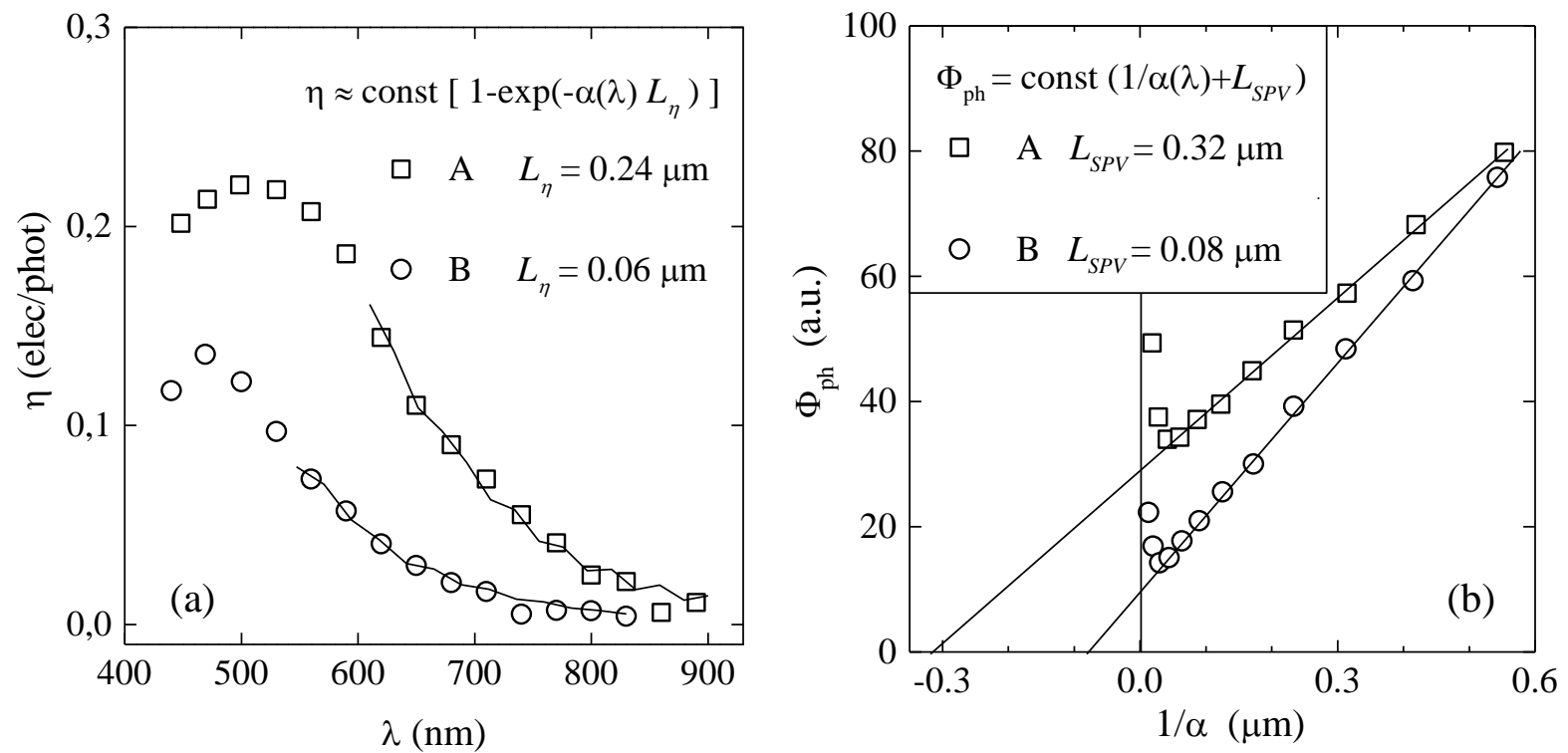

Fig. 2. Collection lengths deduced from the external quantum efficiency $L_{\eta}$ (a) and surface photovoltage measurements $L_{S P V}$ (b) of the Schottky contacts shown in this work. 
In Fig. 2 we compare the collection lengths $L_{\eta}$ and $L_{S P V}$ deduced from external quantum efficiency and surface photovotage measurements for both Schottky contacts. The $A$ contact presents clearly higher collection lengths. This agrees with its much lower concentration of localised states in the bandgap acting as recombination centres. These results correlate with the enhanced photovoltaic performance observed in solar cells whose active layers were grown faster. However, though the same trend is observed, deduced surface photovoltage collection lengths are higher than those calculated from the external quantum efficiency, i.e., $L_{S P V}>L_{\eta}$. It has been previously reported that if the surface photovoltage method is applied to thin film Schottky contacts of low mobility semiconductors, overestimated collection lengths could be obtained[8]. The straightforward graphical method is only valid if the collection length is much longer than the space region width, but this condition is often not fulfilled in the case of low mobility semiconductors as nc-Si:H.

\section{Conclusions}

By increasing the deposition rate, the concentration of localised states in the bandgap of nc-Si:H samples could be lowered in more than one order of magnitude. By contrast, this result did not correlate with the subgap optical absorption and only slight differences were observed in the microstructure of the samples. Therefore, these localised states should be due to the undesired incorporation of contaminants not resolvable by subgap optical absorption due to its shallow position in the gap. These contaminants could be attributed to gas phase impurities (nitrogen, oxygen...) degassed from inner parts of the deposition chamber and metallic impurities (tungsten, copper...) evaporated from the filament.

The external quantum efficiency and surface photovoltage of Schottky contacts are very useful measurements to evaluate the quality of the electronic transport in the direction perpendicular to the substrate for nc-Si:H thin films. These measurements allow to deduce effective transverse collection lengths which correlate with the photovoltaic performance of solar cells. However, the collection lengths evaluated by the surface photovoltage method result overestimated in the case of low mobility semiconductors if the simple graphical method is directly applied.

\section{References}

[1] R.E.I. Schropp, J. K. Rath, IEEE Transactions on Electron Devices, 46, 2069-2071 (1999)

[2] J. Kocka, A. Fejfar, H. Stuchlikova, B. Rezek, A. Poruba, M. Vanecek, P. Torres, J. Meier, N. Wyrsch, A. Shah, A. Matsuda, Proc. of the $2^{\text {nd }}$ WC PVSEC in Vienna, 1, 785-788 (1998)

[3] D. Peiro, Ph.D Thesis, University of Barcelona (1999)

[4] E.H. Rhoderick, Metal-Semiconductor Contacts, Clarendon Oxford (1978)

[5] A.M. Goodman, J. Appl. Phys., 32, 2550-2552 (1961)

[6] C. Voz, M. Fonrodona, D. Soler, J.M. Asensi, J. Bertomeu, J. Andreu, Proc. of the $16^{\text {th }}$ EC PVSEC in Glasgow, in press (2000)

[7] W.B. Jackson, N.M. Johnson, D.K. Biegelsen, Appl. Phys. Lett., 43, 195-197 (1983)

[8] A. R. Moore, J. Appl. Phys., 54, 222-228 (1983) 